\title{
Modeling of Quinoacridinium Derivatives as Antitumor Agents using a QSAR analysis
}

\section{Ruslin Hadanu}

Department of Chemistry Education, Faculty of Teacher Training and Education Science, Universitas Sembilanbelas November Kolaka, Jl. Pemuda No. 339 Kolaka, Sulawesi Tenggara 93517, Indonesia

\begin{tabular}{|c|c|}
\hline Info Article & ABSTRACT \\
\hline $\begin{array}{l}\text { Submitted: 29-02-2019 } \\
\text { Revised: 23-05-2019 } \\
\text { Accepted: 19-06-2019 } \\
\text { *Corresponding author } \\
\text { Ruslin Hadanu } \\
\text { Email: } \\
\text { ruslinhadanu@gmail.com }\end{array}$ & 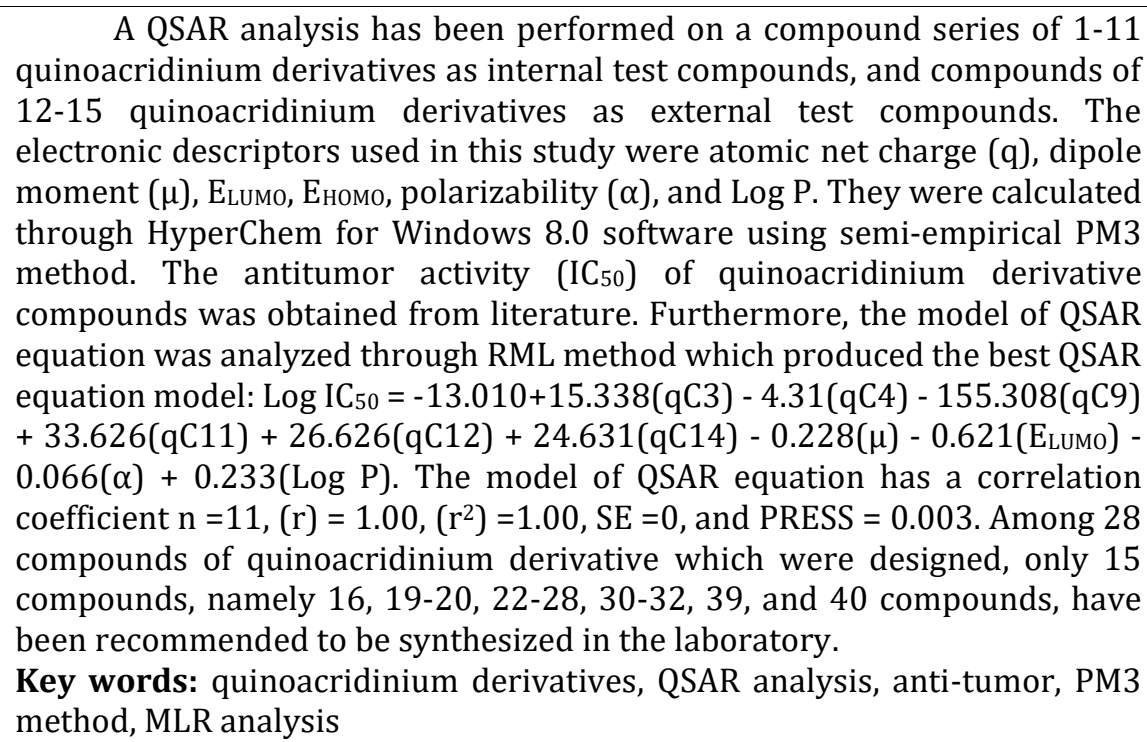 \\
\hline
\end{tabular}

\section{INTRODUCTION}

Tumors and cancers still become dangerous health problem in the world because of their high morbidity and mortality. In 2012, there were 14.1 million new cancer cases, with 8.2 million cancer deaths (Torre et al., 2015). In 2000, productivity costs which was lost due to cancer deaths were 115.8 billion US dollars, and by 2020 , these costs are predicted to reach 147.8 billion US dollars (Bradley et al., 2008). The main problems in a cancer therapy are low selectivity and high toxicity. Therefore, it is necessary to develop antitumor drugs with high selectivity as well as low toxicity, so that the therapy will be more effective. One of the antitumor compounds that has been widely studied and continuously developed by previous researchers is the derivatives of acridinium compounds.

A recent study on acridinium derivatives was conducted in 2008 (Cheng et al., 2008). It was a synthesis on a series of salt-derivative compound with 8,13-dimethylquino[4,3,2-kl]acridinium as an antitumor. Derivatives of the acridinium compounds used as an antitumor have been studied (Cooksonet et al., 2005). Research on the derivatives of acridine compounds has been done by various researchers since 1984 . They isolated acridine compounds from plant species, Lactariusnecator (Agaricales) (Fugmann et al., 1984). Furthermore, after 13 years later, the ring of acridine compounds was successfully synthesized and antitumor activity was tested (Hagan et al., 1997). After that in 1998, several researchers conducted a study to convert 9-azidoacridine to 7H-pyrido [4,3,2-kl]acridine compound and succeeded in changing the pyridine ring to $7 \mathrm{H}$ pyrido[4,3,2-kl]acridine compound to increase the antitumor derivative activity of acridine compounds (Hagan et al., 1998; Julino \& Stevens, 1998). In 2000, the ring of acridine compound was developed into tetra-, penta-, and hexacyclic rings in a heteroaromatic system through a cyclical process of 9-anilinoacridineto enhance antitumor activity of derivatives of acridine compounds which were synthesized in the previous years (Stanslas et al., 2000; Ellis et al., 2001). 
Research on the derivatives of acridine compounds continues being developed by researchers. Some of them are the synthesis of acridine polycyclic compounds, i.e. 8,13-diethyl6-methylquino[4,3,2-kl]acridinium iodide and 3,11-difluoro-6,8,13-trimethyl-8H-quino[4,3,2-

$\mathrm{kl}$ ]acridinium methosulfate (Missailidis et al., 2002; Leonetti, 2004). The synthesis of the acrylic polycyclic derivative was intended to increase the antitumor activity and the solubility of the compound (Missailidis et al., 2002; Leonetti, 2004). Efforts to obtain a more active antitumor through the derivatives of acridine compounds were continued by researchers through the synthesis of more polar and complex compounds using the alkylation method of polycyclic acridine compounds (Cookson et al., 2005). The development of antitumor compounds from polycyclic acridine has been carried out (Cheng et al., 2008). It is a salt compound of 8,13dimethylquino[4,3,2-kl]acridinium and has an antitumor activity ( $\mathrm{IC}_{50}$ ) around 0.21 to $2.00 \mu \mathrm{M}$. The salt compound which has the highest activity is derivatives of 8,13-dimethylquino[4,3,2$\mathrm{kl}$ ]acridinium which is bound up both with anionic iodine and an ester functional group on C2 atom of such cyclic compound ring. Antitumor activity of salt derivatives 8,13-dimethylquino[4,3,2$\mathrm{kl}$ ]acridinium compound is still low when it is compared with antitumor compounds that have been circulating in the market (Cheng et al., 2008). Based on the results of the research above, the efforts to develop antitumor compounds from other compounds have also been developed recently, such as o-isoselenazolon, and galliumpyridine complexes known as metal-based drugs with a very high anticancer activity (Schmitt \& Dou, 2013; Florea \& Büsselberg, 2011; Luo et al., 2012). However, the development of an anticancer compound from heavy metals causes many metabolic risks to the human body. Other studies on anticancer and antitumor have also been done by many researchers (Miladiyah et al., 2016; Hosny et al., 2012; Alam et al., 2016; Reddy et al., 2012; Bladt et al., 2013; Heliawati et al., 2015; Noolvi and Patel, 2013; Deep et al., 2016; Shelton et al., 2016; Tripodi et al., 2012; Su et al., 2011). They consecutively isolated and modified the chemical structures on the following compounds: (1) derivatives of benzoylphenylurea; (2) derivatives of $N$-benzoyl cephalexin; (3) derivatives of benzoyl paracetamol mercaptotriazoles; (4) derivatives of coumarin and quinolinyl; (5) sesquiterpenecoumarin; (6) derivatives of tri-terpenoide saponin; (7) extracts of Acalyphaindica leaf; (8) derivatives of polyketide; (9) extracts of Scaevolaspinescens; (10) extracts from the Coryphautan Lamk; (11) derivatives of polyketide; (12) derivatives of 2,3,7-trisubstituted quinazoline; (13) derivatives of 2-azetidinone; (14) derivatives of glutathione; (15) derivatives of nucleoside and nucleotide; (16) derivatives of 1,4-diaryl-2azetidinones; and (17) xanthone derivative compounds (Miladiyah et al., 2016; Hosny et al., 2012; Alam et al., 2016; Reddy at al., 2012; Bladt et al., 2013; Heliawati et al., 2015; Noolvi \& Patel, 2013; Deep et al., 2016; Shelton et al., 2016; Tripodi et al., 2012; Su et al., 2011). The antitumor activities of all compounds afore mentioned before are still low when they are compared with antitumor compounds that have been circulating in the market. All compounds which were isolated and modified generally have a functional group of primary amine $\left(-\mathrm{NH}_{2}\right)$. Based on the results of the research above, to design the structure of antitumor or anticancer molecules, it is necessary to make a model of anticancer molecules whose structures are cyclic compounds and have functional groups of amine $\left(-\mathrm{NH}_{2}\right)$. In this study it was attempted to synthesize quinoacridinium derivative compounds that have an amine functional group, so that they are antitumor compounds that meet this criterion.

Based on the explanation above, the effort to find an antitumor compound from the 8,13dimethylquino[4,3,2-kl]acridinium which has a functional group $-\mathrm{NH}_{2}$ is still very potential to be a candidate for antitumor drugs which are expected to have activity high and friendly to the body. Efforts to develop an antitumor drug from 8,13dimethylquino[4,3,2-kl]acridinium derivatives need to be done through a computational chemistry approach with a Quantitative Structure-Activity Relationship (QSAR) analysis to obtain more active and body-friendly compounds. The QSAR analysis is one of the latest methods in the phases of developing new drugs. The results of QSAR analysis are generally used as a guide to design new drugs theoretically. In this study, the results of QSAR analysis are used as guidance in designing compounds of 8,13dimethylquino[4,3,2-kl] acridinium derivatives that are potentially to be antitumor and have never been previously synthesized. The QSAR analysis approach as an effort to design drug compounds is very important because it minimizes the use of chemicals and energy. It also saves time because it can avoid trial and error experiments in laboratory. 
However, it can still provide a relatively high level of confidence (Hadanu and Syamsudin, 2013). The QSAR analysis aims to find an empirically consistent relationship between the molecular properties and the biological activity of a series of homologous structural compounds of the drug. The QSAR study began to grow rapidly after1960. It was pioneered by Corwin Hansch who connected chemical structures with drug biology activities through common chemical-physical properties such as fat solubility, ionization degree, and molecular size. Later on in the last few decades, it was developed more intensively into a quantitative relationship between biological activity and various chemical-physical parameters such as net atomic charge, Eцuмо-Еномо, solubility in fat (polarisabilities), solubility in water and alcohol (Log P), molecular size, hydrophobic parameters, electronics and sterics in a series of molecules (Hadanu and Syamsudin, 2013). The relationship between physical as well as chemical properties and biological activity is proposed by (Ferguson et al., 1997) with an equation that can be used to relate some activity data with the following parameters:

$\log \mathrm{BR}=\mathrm{f}\left(\mathrm{P}_{1}, \mathrm{P}_{2}, \mathrm{P}_{3}\right)$

$\mathrm{BR}$ (Biological Response) is a biological activity or biological response as an algebraic function of 3 parameters $P_{1}, P_{2}$, and $P_{3}$ which is the nature of the structure under investigation. The development of QSAR analysis in the next period uses the net charge of the atom as an estimator and expressed by:

$\log \mathrm{BR}=\sum_{i} P_{i} q_{i}+C$

$\mathrm{P}_{\mathrm{i}}$ is the fitting parameter for $\mathrm{i}^{\mathrm{th}}$ atom, and $\mathrm{q}^{\mathrm{i}}$ is the net charge of the atoms and other parameters for the $i^{\text {th }}$ atom and $\mathrm{C}$ is constant. In the development of subsequent research, QSAR study is very helpful in the search for new drugs with greater activity, higher selectivity, toxicity or minor side effects, and greater comfort. In addition, using the QSAR equation model can save more money because to get a new drug with high activity, the experimental factor can be minimized as much as possible (Hadanu et al., 2015).

Some attempts at cancer treatment have been performed in various ways such as surgery, radiation, anticancer drug treatment or chemotherapy. However, these efforts have not achieved satisfying results; even the effects of surgical failure can cause cancer to spread to other parts of the body with severe conditions (Nugraha et al., 2018). This encourages the development of new medicine from 8,13-dimethylquino[4,3,2kl]acridinium compound which is more active, friendly to the body, and expectedly to have a good therapeutic effect.

\section{MATERIAL AND METHODS}

The materials used in this study were quinoacridinium derivative compounds that have been synthesized by Cheng et al., (2008). Inhibition Concentration ( $\mathrm{IC}_{50}$ ) was used as the dependent variable (Table I and II) (Cheng et al., 2008).

\section{Instrumentation}

In this study, the tools used for QSAR test were computer hardware devices namely a Sony Vaio Laptop with Intel® Dual Core Processor 2.20 GHz; 1 GHz RAM, and HDD 250 GB. Meanwhile, the software used in this study is HyperChem 8.0 for Windows for optimization purposes of 3D structure and geometry optimization of the chemical structure of tested compounds (compounds 1-11), external test compounds (compounds 12-15) and the structure of quinoacridinium derivative compounds (compound 16-43). For statistical analysis to obtain the QSAR equation, SPSS 19.0 for Windows was used.

\section{Calculating the descriptors}

Internal fitting compounds (Table I), external fitting compounds (Table II), and modeling compounds (Table V) were constructed respectively in three-dimensional (3D) structures with the HyperChem 8.0 for Windows programming package. Furthermore, the geometric structures of all fitting compounds and model compounds were optimized to obtain more stable structural conformation using the semi-empirical PM3 method. This method is chosen because semi-empirical methods can be performed descriptor calculations quickly and accurately. The semi-empirical method is faster than the ab-initio method although it is less accurate and the semi-empirical method is more accurate than the AM1 method even though it is slower than the AM1 method. In addition, semi-empirical methods are methods that use experimental and theoretical data sets. When viewed with the molecular structure used, semi-empirical methods are suitable, because the quinoacridinium molecule have a medium molecular weight and functional groups $-\mathrm{NH}_{2},-\mathrm{NHR}$, and $-\mathrm{NR}_{2}$. 
Table I. Chemical structure and activity data of antitumor compounds of quinoacridinium derivatives obtained from Cheng et al., (2008)

\begin{tabular}{|c|c|c|c|c|c|c|c|c|c|c|}
\hline Compounds & $\mathbf{R}^{2}$ & $\mathbf{R}^{3}$ & $\mathbf{R}^{6}$ & $\mathbf{R}^{8}$ & $\mathbf{R}^{10}$ & $R^{11}$ & $\mathbf{R}^{13}$ & $\mathbf{X}$ & $\begin{array}{c}\begin{array}{c}\mathrm{IC}_{50} \\
(\mu \mathrm{M})\end{array} \\
\end{array}$ & $\begin{array}{l}\text { Log } \\
\text { IC }_{50} \\
\end{array}$ \\
\hline 1 & $\mathrm{H}$ & $\mathrm{H}$ & $\mathrm{CH}_{3}$ & $\mathrm{C}_{2} \mathrm{H}_{5}$ & $\mathrm{H}$ & $\mathrm{H}$ & $\mathrm{C}_{2} \mathrm{H}_{5}$ & $\mathrm{I}^{-}$ & 2.00 & 0.30 \\
\hline 2 & $\mathrm{H}$ & $\mathrm{CH}_{3}$ & $\mathrm{CH}_{3}$ & $\mathrm{CH}_{3}$ & $\mathrm{H}$ & $\mathrm{CH}_{3}$ & $\mathrm{CH}_{3}$ & $\mathrm{H}_{3} \mathrm{COSO}_{3}^{-}$ & 0.25 & -0.60 \\
\hline 3 & $\mathrm{H}$ & $\mathrm{H}$ & $\mathrm{CH}_{3}$ & $\mathrm{CH}_{3}$ & $\mathrm{H}$ & $\mathrm{H}$ & $\mathrm{CH}_{3}$ & $\mathrm{H}_{3} \mathrm{COSO}_{3}^{-}$ & 0.74 & -0.13 \\
\hline 4 & $\mathrm{H}$ & $\mathrm{H}$ & $\mathrm{H}$ & $\mathrm{CH}_{3}$ & $\mathrm{H}$ & $\mathrm{H}$ & $\mathrm{CH}_{3}$ & $\mathrm{I}^{-}$ & 1.55 & 0.19 \\
\hline 5 & $\mathrm{NHCOCH}_{3}$ & $\mathrm{H}$ & $\mathrm{H}$ & $\mathrm{CH}_{3}$ & $\mathrm{H}$ & $\mathrm{H}$ & $\mathrm{CH}_{3}$ & $\mathrm{I}^{-}$ & 0.38 & -0.42 \\
\hline 6 & $\mathrm{NHCOCF}_{3}$ & $\mathrm{H}$ & $\mathrm{H}$ & $\mathrm{CH}_{3}$ & $\mathrm{H}$ & $\mathrm{H}$ & $\mathrm{CH}_{3}$ & $\mathrm{I}^{-}$ & 0.31 & -0.51 \\
\hline 7 & $\mathrm{NHCO}\left(\mathrm{CH}_{2}\right)_{4} \mathrm{CO}_{2} \mathrm{CH}_{3}$ & $\mathrm{H}$ & $\mathrm{H}$ & $\mathrm{CH}_{3}$ & $\mathrm{H}$ & $\mathrm{H}$ & $\mathrm{CH}_{3}$ & $\mathrm{I}^{-}$ & 0.21 & -0.68 \\
\hline 8 & $\mathrm{~N}\left(\mathrm{CH}_{3}\right) \mathrm{SO}_{2} \mathrm{C}_{6} \mathrm{H}_{4}-p-\mathrm{F}$ & $\mathrm{H}$ & $\mathrm{H}$ & $\mathrm{CH}_{3}$ & $\mathrm{H}$ & $\mathrm{H}$ & $\mathrm{CH}_{3}$ & $\mathrm{I}^{-}$ & 0.73 & -0.14 \\
\hline 9 & $\mathrm{H}$ & $\mathrm{NHCOCH}_{3}$ & $\mathrm{H}$ & $\mathrm{CH}_{3}$ & $\mathrm{H}$ & $\mathrm{H}$ & $\mathrm{CH}_{3}$ & $\mathrm{I}^{-}$ & 0.41 & -0.39 \\
\hline 10 & $\mathrm{H}$ & $\mathrm{Cl}$ & $\mathrm{OCH}_{3}$ & $\mathrm{CH}_{3}$ & $\mathrm{H}$ & $\mathrm{H}$ & $\mathrm{CH}_{3}$ & $\mathrm{I}^{-}$ & 0.28 & -0.55 \\
\hline 11 & $\mathrm{H}$ & $\mathrm{CH}=\mathrm{CHCON}\left(\mathrm{CH}_{2} \mathrm{CH}_{2}\right)_{2} \mathrm{O}$ & $\mathrm{H}$ & $\mathrm{CH}_{3}$ & $\mathrm{H}$ & $\mathrm{H}$ & $\mathrm{CH}_{3}$ & $\mathrm{I}^{-}$ & 0.37 & -0.43 \\
\hline
\end{tabular}

Table II. The chemical structure of external standard compounds of quinoacridinium derivatives from Cheng et al., (2008).

\begin{tabular}{|c|c|c|c|c|c|c|c|c|c|c|}
\hline Compounds & $\mathbf{R}^{2}$ & $\mathbf{R}^{3}$ & $\mathbf{R}^{6}$ & $\mathbf{R}^{8}$ & $\mathbf{R}^{10}$ & $\mathbf{R}^{11}$ & $\mathbf{R}^{13}$ & $\mathbf{X}^{-}$ & $\begin{array}{c}I_{50} \\
(\mu \mathrm{M})\end{array}$ & $\log \mathrm{IC}_{50}$ \\
\hline 12 & $\mathrm{H}$ & $\mathrm{F}$ & $\mathrm{CH}_{3}$ & $\mathrm{CH}_{3}$ & $\mathrm{H}$ & $\mathrm{F}$ & $\mathrm{CH}_{3}$ & $\mathrm{H}_{3} \mathrm{COSO}_{3}^{-}$ & 0.33 & -0.48 \\
\hline 13 & $\mathrm{NH}_{2}$ & $\mathrm{H}$ & $\mathrm{H}$ & $\mathrm{CH}_{3}$ & $\mathrm{H}$ & $\mathrm{H}$ & $\mathrm{CH}_{3}$ & $\mathrm{I}^{-}$ & 1.86 & 0.27 \\
\hline 14 & $\mathrm{NHCOC}\left(\mathrm{CH}_{3}\right)_{3}$ & $\mathrm{H}$ & $\mathrm{H}$ & $\mathrm{CH}_{3}$ & $\mathrm{H}$ & $\mathrm{H}$ & $\mathrm{CH}_{3}$ & $\mathrm{I}^{-}$ & 0.23 & -0.64 \\
\hline 15 & $\mathrm{H}$ & $\mathrm{Cl}$ & $\mathrm{H}$ & $\mathrm{CH}_{3}$ & $\mathrm{H}$ & $\mathrm{H}$ & $\mathrm{CH}_{3}$ & $\mathrm{I}^{-}$ & 0.26 & -0.59 \\
\hline
\end{tabular}

The structural optimization was performed by using Polak-Ribiere algorithm optimization method with RMS value $=0.001 \mathrm{kcal} /(\AA \AA \mathrm{mol})$, which was recorded through the single point menu. Via the data fromthe single point menu, the electronic parameters such as the net atomic charge, the dipole moment $(\mu)$, the polarization $(\alpha)$, and the Log P were obtained. Meanwhile, the E Eumo and the Еномо were obtained from compute and orbital menus which were presented (Table III and IV). The selection of descriptor type was adjusted to the type of descriptor used by Motta et al., (2006) in conducting QSAR analysis. The structure of the quinoacridinium derivative compounds as the study material was calculated by involving an ion counter of salt.

\section{QSAR equation analysis by using a linear regression method}

The dependent variable in this study was the antitumor activity with $\mathrm{IC}_{50}$ value based on experiment (Table I), while the independent 
Table III. Log IC 50 experiments and Log IC $\mathrm{I}_{50}$ calculated of external test compounds

\begin{tabular}{ccc}
\hline Compounds & Log IC $\mathbf{5 0}_{\mathbf{0}}$ experiments & Log IC $\mathbf{5 0}_{\mathbf{5}}$ calculated \\
\hline 12 & -0.48 & -0.359 \\
13 & 0.27 & 0.166 \\
14 & -0.64 & -0.602 \\
15 & -0.59 & -0.589 \\
\hline
\end{tabular}

Table IV. Descriptors/independent variables used for QSAR analysis of antitumor compounds of quinoacridinium derivatives calculated by the semi-empirical PM3 method

\begin{tabular}{|c|c|c|c|c|c|c|c|c|c|c|c|c|c|}
\hline \multirow{2}{*}{$\begin{array}{c}\text { Comp. } \\
\text { Number }\end{array}$} & \multicolumn{13}{|c|}{ Atomic net charges (Coulomb) } \\
\hline & qC1 & $\mathbf{q C 2}$ & qC3 & qC4 & qC5 & qC6 & qC7 & qN8 & qC9 & qC10 & qC11 & $q \mathrm{C12}$ & $\mathrm{qN} 1$ \\
\hline 1 & -0.075 & -0.060 & -0.077 & -0.083 & -0.160 & 0.033 & -0.193 & 0.200 & -0.179 & -0.015 & -0.112 & 0.03 & 0.200 \\
\hline 2 & -0.056 & & & 5 & -0.153 & 0.023 & -0.187 & 0.188 & -0.165 & -0.034 & -0.062 & t & 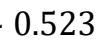 \\
\hline 3 & -0.069 & & & & -0.155 & 0.024 & -0.189 & 0.188 & -0.182 & -0.025 & -0.114 & -0.073 & 491 \\
\hline 4 & -0.019 & -0 & & & & -0.015 & & 0.170 & -0.170 & -0.030 & -0.124 & -0.046 & 0.573 \\
\hline 5 & -0 . & & & & & & & 0.194 & & -0.017 & -0 & & \\
\hline 6 & & & & & & & & 0.175 & -0.170 & -0 . & & & \\
\hline 7 & & & & & & & & 0.187 & -0.173 & -0.031 & -0.115 & -4 & 420 \\
\hline 8 & -0.2 & & & & & & & 0.189 & -0.176 & & & 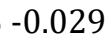 & \\
\hline 9 & & & & & & & & & & & & & \\
\hline 10 & & & & & & & & & & & & & \\
\hline 11 & & 74 & -0.038 & -0.064 & 0.139 & -0.020 & -1 & 0.189 & -0.172 & -0.032 & -0.111 & -0.00 & \\
\hline \multirow{2}{*}{$\begin{array}{c}\text { Comp. } \\
\text { Number }\end{array}$} & \multicolumn{8}{|c|}{ Atomic net charges (Coulomb) } & \multirow{2}{*}{$\begin{array}{c}\mu \\
\text { (Debye) }\end{array}$} & \multirow{2}{*}{$\begin{array}{c}\text { Elumo } \\
\text { (ev) }\end{array}$} & \multirow{2}{*}{$\begin{array}{c}\text { Еномо } \\
\text { (ev) }\end{array}$} & \multirow{2}{*}{$\begin{array}{c}\alpha \\
\left(\AA^{3}\right)\end{array}$} & \multirow{2}{*}{$\log P$} \\
\hline & qC14 & $\mathrm{qC15}$ & qC16 & qC17 & qC18 & qC19 & $q C 20$ & qC21 & & & & & \\
\hline 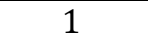 & -0.126 & -0.052 & 0.067 & 0.073 & -0.145 & -0.1 & -0.019 & 0.012 & 20.946 & -2.563 & -4.236 & 46.27 & 2 \\
\hline 2 & & & & 0.017 & & & & 0. & 287 & & & 45.49 & 94 \\
\hline 3 & -0 & & 0. & 0.0 & & & & & & & & 2 & \\
\hline 4 & -0.124 & -0.033 & 0.056 & 0.000 & -0 . & -0 . & -0 . & -0.010 & 13.352 & -2.078 & -5.712 & 40.77 & 1.28 \\
\hline 5 & -0.115 & -0.079 & 0.070 & 0.049 & -0.1 & -0. & -0.1 & -0.003 & 15.507 & -2.541 & -4.590 & 45.88 & -0.61 \\
\hline 6 & -0.098 & -0.049 & 0.060 & 0.018 & -0.119 & -0.124 & -0.024 & -0.008 & 14.899 & -2.311 & -5.323 & 45.60 & 0.50 \\
\hline 7 & -0.094 & & 0.048 & -0.005 & -0.1 & & & -0.015 & 12.513 & -2.478 & -5.327 & 55.77 & 0.00 \\
\hline 0 & -0.0 & & 0.077 & 0.0 & -0 & & & & & -2.456 & & 54.66 & -0.56 \\
\hline 0 & -0. & -0. & 0.028 & -0.0 & -0. & -0. & & -0.017 & 14.072 & -2.604 & -5 . & 45.88 & -0.61 \\
\hline 10 & -0. & -0. & 0.087 & 0.0 & -0.1 & -0. & -0 & 0.031 & 15.096 & -2.151 & -5.098 & 45.17 & 0.06 \\
\hline 11 & -0.127 & -0.033 & 0.035 & -0.007 & -0.094 & -0.108 & -0.030 & -0.017 & 15.213 & -2.558 & -5.367 & 54.72 & 0.36 \\
\hline
\end{tabular}

variables used were net atomic charges, dipole moment $(\mu)$, Eцимо, Еномо, polarizability $(\alpha)$, and Log P (Tables III and IV). All variables were analyzed using enter method through MLR to find out which sequence of independent variables influenced the antitumor activity value of quinoacridinium derivative compounds. The result generated QSAR equations as well as statistical parameters such as $r, r^{2}$, SE, and PRESS values. In addition to the statistical parameters, the calculation result also obtained the constant value and the coefficient value of each independent variable involved in the equation result. The obtained coefficient value was used to calculate the theoretical antitumor activity ( $\mathrm{IC}_{50}$ theoretical value) toward quinoacridinium derivative compounds. Furthermore, after finding out the square difference betweenthe $\mathrm{IC}_{50}$ experimental value andthe $\mathrm{IC}_{50}$ theoretical value, the PRESS value can be calculated to know the quality and prediction ability of the best equation of the QSAR model.

\section{Design of new antitumor molecules}

The design of new antitumor drug molecules in this study was aimed to discover novel 
Table VI. The New designed quinoacridinium derivatives as antitumor compounds

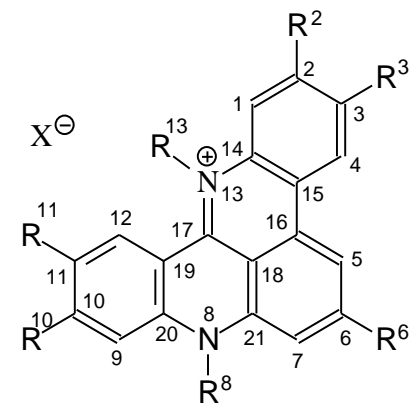

\begin{tabular}{|c|c|c|c|c|c|}
\hline \multirow{2}{*}{ Compounds } & \multicolumn{5}{|c|}{ Substituents } \\
\hline & $\mathbf{R}^{2}$ & $\mathbf{R}^{3}$ & $\mathbf{R}^{6}$ & $\mathbf{R}^{8}$ & $\mathbf{R}^{10}$ \\
\hline 16 & $\mathrm{H}$ & $\mathrm{Cl}$ & $\mathrm{CH}_{3}$ & $\mathrm{CH}_{3}$ & $\mathrm{H}$ \\
\hline 17 & $\mathrm{NHCH}_{3}$ & $\mathrm{H}$ & $\mathrm{H}$ & $\mathrm{CH}_{3}$ & $\mathrm{H}$ \\
\hline 18 & $\mathrm{~N}\left(\mathrm{CH}_{3}\right)_{2}$ & $\mathrm{H}$ & $\mathrm{H}$ & $\mathrm{CH}_{3}$ & $\mathrm{H}$ \\
\hline 19 & $\mathrm{NHCOC}_{4} \mathrm{H}_{9}$ & $\mathrm{H}$ & $\mathrm{H}$ & $\mathrm{CH}_{3}$ & $\mathrm{H}$ \\
\hline 20 & $\mathrm{NHCOC}_{11} \mathrm{H}_{23}$ & $\mathrm{H}$ & $\mathrm{H}$ & $\mathrm{CH}_{3}$ & $\mathrm{H}$ \\
\hline 21 & $\mathrm{NHCO}_{2} \mathrm{C}_{2} \mathrm{H}_{5}$ & $\mathrm{H}$ & $\mathrm{H}$ & $\mathrm{CH}_{3}$ & $\mathrm{H}$ \\
\hline 22 & NHCOPh & $\mathrm{H}$ & $\mathrm{H}$ & $\mathrm{CH}_{3}$ & $\mathrm{H}$ \\
\hline 23 & $\mathrm{H}$ & $\mathrm{H}$ & $\mathrm{OCH}_{3}$ & $\mathrm{CH}_{3}$ & $\mathrm{Cl}$ \\
\hline 24 & $\mathrm{H}$ & $\mathrm{H}$ & $\mathrm{OCH}_{3}$ & $\mathrm{CH}_{3}$ & $\mathrm{CH}=\mathrm{CHCON}\left(\mathrm{CH}_{2} \mathrm{CH}_{2}\right)_{2} \mathrm{O}$ \\
\hline 25 & $\mathrm{H}$ & $\mathrm{H}$ & $\mathrm{OCH}_{3}$ & $\mathrm{CH}_{3}$ & $\mathrm{CH}=\mathrm{CHCO}_{2} \mathrm{CH}_{3}$ \\
\hline 26 & $\mathrm{H}$ & $\mathrm{H}$ & $(\mathrm{CH} 2)_{3} \mathrm{OCOCH}_{3}$ & $\mathrm{CH}_{3}$ & $(\mathrm{CH} 2)_{3} \mathrm{OCOCH}_{3}$ \\
\hline 27 & $\mathrm{H}$ & $\mathrm{Cl}$ & $\mathrm{CH}=\mathrm{CHCON}\left(\mathrm{CH}_{2} \mathrm{CH}_{2}\right)_{2} \mathrm{O}$ & $\mathrm{CH}_{3}$ & $\mathrm{H}$ \\
\hline 28 & $\mathrm{H}$ & $\mathrm{CH}=\mathrm{CHCON}\left(\mathrm{CH}_{2} \mathrm{CH}_{2}\right)_{2} \mathrm{O}$ & $\mathrm{OCH}_{3}$ & $\mathrm{CH}_{3}$ & $\mathrm{H}$ \\
\hline 29 & $\mathrm{H}$ & $\mathrm{CH}=\mathrm{CHCON}\left(\mathrm{CH}_{2} \mathrm{CH}_{2}\right)_{2} \mathrm{O}$ & $\left(\mathrm{CH}_{2}\right)_{3} \mathrm{OCOCH}_{3}$ & $\mathrm{CH}_{3}$ & $\mathrm{H}$ \\
\hline 30 & $\mathrm{H}$ & $\left(\mathrm{CH}_{2}\right)_{3} \mathrm{OCOCH}_{3}$ & $\left(\mathrm{CH}_{2}\right)_{3} \mathrm{OCOCH}_{3}$ & $\mathrm{CH}_{3}$ & $\mathrm{H}$ \\
\hline 31 & $\mathrm{H}$ & $\mathrm{Cl}$ & $\mathrm{CH}_{3}$ & $\mathrm{CH}_{3}$ & $\mathrm{H}$ \\
\hline 32 & $\mathrm{H}$ & $\mathrm{Cl}$ & $\mathrm{CH}_{3}$ & $\mathrm{C}_{2} \mathrm{H}_{5}$ & $\mathrm{H}$ \\
\hline 33 & $\mathrm{~N}\left(\mathrm{C}_{2} \mathrm{H}_{5}\right)_{2}$ & $\mathrm{H}$ & $\mathrm{H}$ & $\mathrm{CH}_{3}$ & $\mathrm{H}$ \\
\hline 34 & $\mathrm{~N}\left(\mathrm{C}_{2} \mathrm{H}_{5}\right)_{2}$ & $\mathrm{H}$ & $\mathrm{H}$ & $\mathrm{C}_{2} \mathrm{H}_{5}$ & $\mathrm{H}$ \\
\hline 35 & $\mathrm{NHCOC}_{3} \mathrm{H}_{7}$ & $\mathrm{H}$ & $\mathrm{H}$ & $\mathrm{C}_{3} \mathrm{H}_{7}$ & $\mathrm{H}$ \\
\hline 36 & $\mathrm{NHCOC}_{2} \mathrm{H}_{5}$ & $\mathrm{H}$ & $\mathrm{H}$ & $\mathrm{C}_{2} \mathrm{H}_{5}$ & $\mathrm{H}$ \\
\hline 37 & $\mathrm{NHCOC}_{11} \mathrm{H}_{23}$ & $\mathrm{H}$ & $\mathrm{H}$ & $\mathrm{C}_{2} \mathrm{H}_{5}$ & $\mathrm{H}$ \\
\hline 38 & NHCOPh & $\mathrm{H}$ & $\mathrm{H}$ & $\mathrm{C}_{2} \mathrm{H}_{5}$ & $\mathrm{H}$ \\
\hline 39 & $\mathrm{H}$ & $\mathrm{H}$ & $(\mathrm{CH} 2)_{3} \mathrm{OCOCH}_{3}$ & $\mathrm{C}_{2} \mathrm{H}_{5}$ & $(\mathrm{CH} 2)_{3} \mathrm{OCOCH}_{3}$ \\
\hline 40 & $\mathrm{H}$ & $\mathrm{Cl}$ & $\mathrm{CH}=\mathrm{CHCON}\left(\mathrm{CH}_{2} \mathrm{CH}_{2}\right)_{2} \mathrm{O}$ & $\mathrm{C}_{2} \mathrm{H}_{5}$ & $\mathrm{H}$ \\
\hline 41 & $\mathrm{H}$ & $\mathrm{CH}=\mathrm{CHCON}\left(\mathrm{CH}_{2} \mathrm{CH}_{2}\right)_{2} \mathrm{O}$ & $\mathrm{OC}_{2} \mathrm{H}_{5}$ & $\mathrm{C}_{2} \mathrm{H}_{5}$ & $\mathrm{H}$ \\
\hline 42 & $\mathrm{H}$ & $\mathrm{CH}=\mathrm{CHCON}\left(\mathrm{CH}_{2} \mathrm{CH}_{2}\right)_{2} \mathrm{O}$ & $\left(\mathrm{CH}_{2}\right)_{3} \mathrm{OCOCH}_{3}$ & $\mathrm{C}_{2} \mathrm{H}_{5}$ & $\mathrm{H}$ \\
\hline 43 & $\mathrm{H}$ & $\left(\mathrm{CH}_{2}\right)_{3} \mathrm{OCOC}_{2} \mathrm{H}_{5}$ & $\left(\mathrm{CH}_{2}\right)_{3} \mathrm{OCO} \mathrm{C}_{2} \mathrm{H}_{5}$ & $\mathrm{C}_{2} \mathrm{H}_{5}$ & $\mathrm{H}$ \\
\hline
\end{tabular}

compounds of quinoacridinium derivatives which have higher antitumor activity than previously synthesized compounds. The molecular design was performed by varying the type and position of substituent or functional group positions in the main framework structure of quinoacridinium derivative compounds. The substituent position was focused on the active center area by considering the feasibility of synthesis in the laboratory. The substituent or functional group is the dominantly responsible atoms of the antitumor activity of the quinoacridinium derivatives. After finding out the quinoacridinium molecular sequence series, the next step is calculating the descriptor of the new compound of modeling design (Table VI and VII) by using the semiempirical method of PM3 using HyperChem 8.0 for Windows. Based on the best QSAR equations, we can calculate the theoretical antitumor activity of the design compounds (Table VI and VII). 
Table VII. Predicted Log IC S0 $_{50}$ calculated using the best QSAR model

\begin{tabular}{|c|c|c|c|c|c|}
\hline \multirow{2}{*}{ Compounds } & \multicolumn{3}{|c|}{ Substituents } & \multirow{2}{*}{ Predicted Log $\mathrm{IC}_{50}$} & \multirow{2}{*}{ Predicted $\mathrm{IC}_{50}$} \\
\hline & $\mathbf{R}^{11}$ & $\mathbf{R}^{13}$ & $\mathbf{X}^{-}$ & & \\
\hline 16 & $\mathrm{Cl}$ & $\mathrm{CH}_{3}$ & $\mathrm{H}_{3} \mathrm{COSO}_{3}^{-}$ & -5.692 & 0.000002 \\
\hline 17 & $\mathrm{H}$ & $\mathrm{CH}_{3}$ & $\mathrm{I}^{-}$ & -0.111 & 0.775149 \\
\hline 18 & $\mathrm{H}$ & $\mathrm{CH}_{3}$ & $\mathrm{I}^{-}$ & 0.080 & 1.201752 \\
\hline 19 & $\mathrm{H}$ & $\mathrm{CH}_{3}$ & $\mathrm{I}^{-}$ & -0.901 & 0.125555 \\
\hline 20 & $\mathrm{H}$ & $\mathrm{CH}_{3}$ & $\mathrm{I}^{-}$ & -0.760 & 0.173970 \\
\hline 21 & $\mathrm{H}$ & $\mathrm{CH}_{3}$ & $\mathrm{I}^{-}$ & -0.315 & 0.484069 \\
\hline 22 & $\mathrm{H}$ & $\mathrm{CH}_{3}$ & $\mathrm{I}^{-}$ & -0.894 & 0.127722 \\
\hline 23 & $\mathrm{H}$ & $\mathrm{CH}_{3}$ & $\mathrm{I}^{-}$ & -1.036 & 0.091970 \\
\hline 24 & $\mathrm{H}$ & $\mathrm{CH}_{3}$ & $\mathrm{I}^{-}$ & -3.928 & 0.000118 \\
\hline 25 & $\mathrm{H}$ & $\mathrm{CH}_{3}$ & $\mathrm{I}^{-}$ & -3.400 & 0.000398 \\
\hline 26 & $\mathrm{H}$ & $\mathrm{CH}_{3}$ & $\mathrm{I}^{-}$ & -1.743 & 0.018086 \\
\hline 27 & $\mathrm{H}$ & $\mathrm{CH}_{3}$ & $\mathrm{I}^{-}$ & -1.254 & 0.055728 \\
\hline 28 & $\mathrm{H}$ & $\mathrm{CH}_{3}$ & $\mathrm{I}^{-}$ & -0.910 & 0.122898 \\
\hline 29 & $\mathrm{H}$ & $\mathrm{CH}_{3}$ & $\mathrm{I}^{-}$ & -0.040 & 0.912860 \\
\hline 30 & $\mathrm{H}$ & $\mathrm{CH}_{3}$ & $\mathrm{I}^{-}$ & -1.460 & 0.034668 \\
\hline 31 & $\mathrm{Cl}$ & $\mathrm{C}_{2} \mathrm{H}_{5}$ & $\mathrm{H}_{5} \mathrm{C}_{2} \mathrm{OSO}_{3}^{-}$ & -5.414 & 0.000004 \\
\hline 32 & $\mathrm{Cl}$ & $\mathrm{C}_{2} \mathrm{H}_{5}$ & $\mathrm{H}_{5} \mathrm{C}_{2} \mathrm{OSO}_{3}-$ & -1.745 & 0.017990 \\
\hline 33 & $\mathrm{H}$ & $\mathrm{C}_{2} \mathrm{H}_{5}$ & $\mathrm{I}^{-}$ & 0.522 & 3.327068 \\
\hline 34 & $\mathrm{H}$ & $\mathrm{C}_{2} \mathrm{H}_{5}$ & $\mathrm{I}^{-}$ & 0.955 & 9.013149 \\
\hline 35 & $\mathrm{H}$ & $\mathrm{C}_{3} \mathrm{H}_{7}$ & $\mathrm{I}^{-}$ & -0.329 & 0.468765 \\
\hline 36 & $\mathrm{H}$ & $\mathrm{C}_{2} \mathrm{H}_{5}$ & $\mathrm{I}^{-}$ & 0.847 & 7.031945 \\
\hline 37 & $\mathrm{H}$ & $\mathrm{C}_{2} \mathrm{H}_{5}$ & $\mathrm{I}^{-}$ & 0.001 & 1.001424 \\
\hline 38 & $\mathrm{H}$ & $\mathrm{C}_{2} \mathrm{H}_{5}$ & $\mathrm{I}^{-}$ & -0.113 & 0.770079 \\
\hline 39 & $\mathrm{H}$ & $\mathrm{C}_{2} \mathrm{H}_{5}$ & $\mathrm{I}^{-}$ & -1.391 & 0.040617 \\
\hline 40 & $\mathrm{H}$ & $\mathrm{C}_{2} \mathrm{H}_{5}$ & $\mathrm{I}^{-}$ & -0.805 & 0.156537 \\
\hline 41 & $\mathrm{H}$ & $\mathrm{C}_{2} \mathrm{H}_{5}$ & $\mathrm{I}^{-}$ & 2.139 & 137.657528 \\
\hline 42 & $\mathrm{H}$ & $\mathrm{C}_{2} \mathrm{H}_{5}$ & $\mathrm{I}^{-}$ & -0.699 & 0.200199 \\
\hline 43 & $\mathrm{H}$ & $\mathrm{C}_{2} \mathrm{H}_{5}$ & $\mathrm{I}^{-}$ & 0.093 & 1.237455 \\
\hline
\end{tabular}

\section{RESULT AND DISCUSSION}

The stages of quantitative relationship analysis of structures carried out in this study were (a) determining series of quinoacridinium compounds which had $\mathrm{IC}_{50}$ values based on experiment in the laboratory Cheng et al., (2008); (b) optimizing the basic structural framework of the most stable quinoacridinium derivative as the initial compound in the process of optimizing the test compound for further analysis; (c) determining descriptor (independent variable); (d) calculating descriptor through optimization structure of tested compounds (optimization of derivative structure of tested compound) (Table I and II); (e) performing correlation analysis between variables through bivariate correlation method; (f) performing multilinear regression analysis to obtain the model of QSAR equation; ( $\mathrm{g}$ ) determining the best QSAR equation model (Hadanu and Syamsudin, 2013; Hadanu et al., 2015) and (h) designing a new compound of quinoacridinium derivatives based on the best QSAR equation model. 


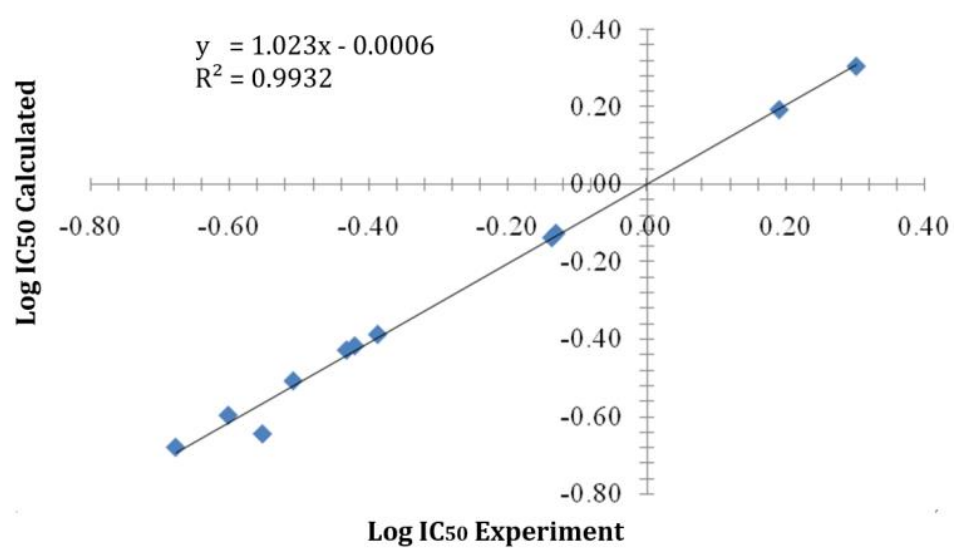

Figure 1. Correlation between $\log \mathrm{IC}_{50}$ calculated from the internal test compound and $\mathrm{Log} \mathrm{IC}_{50}$ experiment

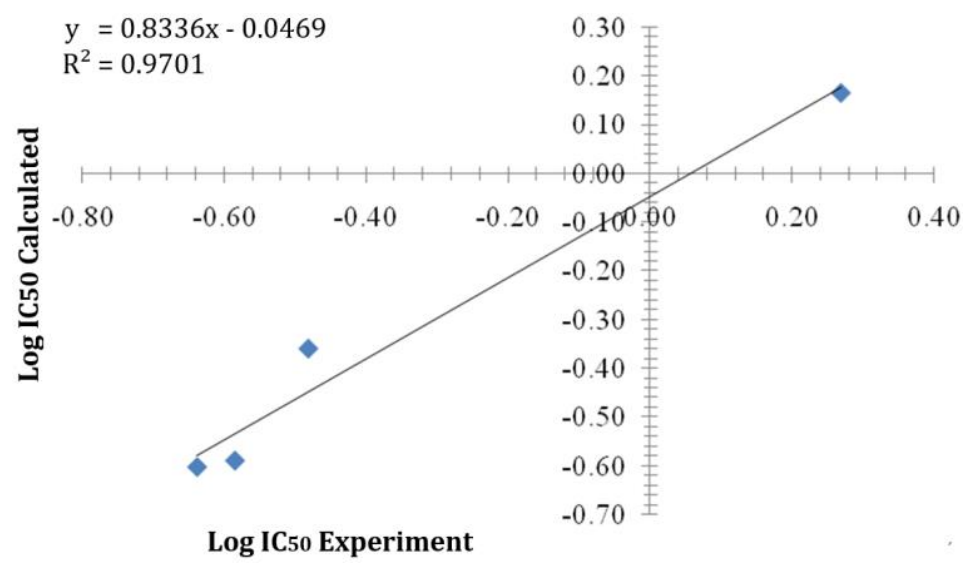

Figure 2. Correlation between $\log \mathrm{IC}_{50}$ of the external test compound and $\mathrm{Log} \mathrm{IC}_{50}$ experiment

The quinoacridinium derivative compounds as the materials in this study has the following requirements: (1) the basic framework structure of the homologous compound should be possessed by all fittings compounds, external test compounds, and the modeled compound; (2) the fitting compound; and (3) the external test compound should have $\mathrm{IC}_{50}$ value which is experimented in a laboratory. The optimization of the structures of all fitting compounds, external test compounds, and the modeled compounds was carried out by the same method to obtain the most stable structural compound with the lowest energy profile. When all of compound structures are at the lowest profile energy, they are in the most stable condition. In that condition, the descriptor (independent variable) required in the next stage of QSAR analysis can be obtained.
By bivariate correlation analysis, it is clear that the variables of atomic net charge, dipole moment $(\mu)$, Еномо-ецuмо, polarisabilities $(\alpha)$, and $\log P$ showed a very close correlation. This is indicated by the absolute value of the correlation which is closer to 1 or -1 . The evidence of such close correlation can be seen in some of the relationships between descriptors that have a high correlation, for example: the correlation between variables QC1-QC2 (-0.893), qC6-qC5 (-0.898), QC2-qC4 (0.774), qC7-qC5 (0.994), qC5-QC10 (0.754), qC6-qC7 (-0.920), QC1-qC15 (0.823), QC2qC15 (-0.865), QC3-qC14 (-0.825), QC3-qC15 (0.831), qC4-qC14 (0.773), qC4-qC15 (-0.897), qN8-qN13 (-0.837), qC5-qC16 (-0.820), qC5-qC17 $(-0.913)$, qC5-qC18 (0.961), qC5-qC19 (0.910), qC6-qC17 (0.724), qC6-qC18 (-0.863), qC6-qC19 $(-0.766), \mathrm{qC} 7-\mathrm{qC} 16 \quad(-0.791), \mathrm{qC} 7-\mathrm{qC} 17$ (-0.890), 
qC7-qC18 (0.963), qC7-qC19 (0.906), QC1- $\alpha$ $(-0.759), q C 5-q C 21(-0.961), q C 5-\mu(-0.764)$, qC6qC21 (0.968), qC6- $\mu$ (0.903), qC7- qC21 (-0.967), $\mathrm{qC7}-\mu$ (-0.778), and qN8-Elumo (-0.768). The negative value of such correlation does not indicate whether the substituent influence on antitumor activity is strong or not, but it only shows the direction of the effect. A negative value indicates correlation of a negative association, which means that the effect of one variable is inversely proportional to other variables. The relationship between the smallest independent variables is the qN8-qN13 (0.001) variable, which indicates that the qN8 variable and qN13 have a weaker relationship than other independent variables. Meanwhile, the relationship between the largest independent variables is variable qC7-qC5 (0.994) which shows that between variables qC7 and $\mathrm{qC} 5$, there is stronger relationship than other independent variables. Based on the bivariate correlation analysis, it can be concluded that between the independent variable and dependent variable, there is a significant relationship. Thus, MLR analysis can be performed on a group of the data in this research.

The MLR analysis on a group of independent variables as descriptor and dependent variable or antitumor activity ( $\log$ IC $_{50}$ ) produced a model of QSAR equation which is the best model of QSAR equation. The best QSAR model built using MLR method is represented by the following equation:

$\log \mathrm{IC}_{50}=-13.010+15.338(\mathrm{qC} 3)-4.31(\mathrm{qC} 4)-$ $155.308(\mathrm{qC} 9)+33.626(\mathrm{qC} 11)+26.626(\mathrm{qC} 12)+$ $24.631(\mathrm{qC} 14)-0.228(\mu)-0.621\left(\right.$ ELMO $\left._{\text {Lu }}\right)-0.066(\alpha)+$ $0.233(\log P)$

The statistical significance of the best QSAR models has coefficient correlation $n=11,(r)=1.00$, $\left(\mathrm{r}^{2}\right)=1.00, \mathrm{SE}=0$, and PRESS $=0.003$.

To test the accuracy of the model of the obtained QSAR equation, it is necessary to calculate the $\mathrm{IC}_{50}$ prediction value of the internal test compound (Table I). The proof of the accuracy of the model of QSAR equation can be seen on the graph of $\log \mathrm{IC}_{50}$ value of prediction with an $\mathrm{IC}_{50}$ value of the experimental internal test (compounds 1-11) (Figure 1). The graph shows the relationship between the $\log \mathrm{IC}_{50}$ prediction value of the internal test compound and $\mathrm{Log} \mathrm{IC}_{50}$ experiment with obtained value $r^{2}=0.993$. The value of $r^{2}$ is close to 1 , which indicates that the internal test model of QSAR equation has a very high level of trust.
To test the validity of the model of QSAR equation, a validity test has been performed by using external test compounds (compounds 1215). The external test compound is a quinoacridinium compound derivative which has been known as $\mathrm{IC}_{50}$ experimental value, but it is not included in the calculation process in determining QSAR equation model. This is intended to validate the QSAR model more accurately since it is validated with internal test compounds and external test compounds. The $\mathrm{IC}_{50}$ value of external compound test (Table III).

The graphic of correlation between $\mathrm{Log}_{\mathrm{IC}} \mathrm{C}_{50}$ calculated and Log $\mathrm{IC}_{50}$ experiments value has $r^{2}=0.9701$ (Figure 2). It shows that the QSAR equation model obtained in this research is the best QSAR equation for determining the $\mathrm{IC}_{50}$ value of compound derivative quinoacridinium. The high value of $\mathrm{r}^{2}$ (close to 1 ) displayed in the correlation graph between Log $\mathrm{IC}_{50}$ calculated from the external test compound and the Log $\mathrm{IC}_{50}$ value of the experiments reinforces the QSAR model as an equation with high validity.

Based on the value of $r^{2}=0.9701$ obtained from the correlation between $\log \mathrm{IC}_{50}$ of calculated from external test compound and Log $\mathrm{IC}_{50}$ experiment, it proves that the relationship between calculated $\mathrm{IC}_{50}$ value of external test compound and Log IC $_{50}$ value of experiment is very strong. Thus, it can be concluded that the model of QSAR equation obtained by using MLR analysis is very significant to determine the value of $\log \mathrm{IC}_{50}$ compounds models of quinoacridinium derivatives.

\section{Design of new antitumor quinoacridinium derivative compounds}

The molecular design of the quinoacridinium derivative remains concerned with the active side of the homologous and atomic frameworks or the functional groups bound to the basic framework structure of the compound. The differences of atoms or functional groups bound to the basic frameworks of homologous compounds of quinoacridinium derivatives may cause different charges of atoms and differences in physical and chemical properties. In addition, it also causes differences in antitumor activity of such compounds (Hadanu et al., 2015). The obvious that compounds which have different structures can produce different antitumor activity (Table I-V). Based on this fact, it can be concluded that different structures have electronic properties and molecular properties (different net charge of atoms and other descriptors). Compounds that have 
Table V. Descriptors/independent of external standard for QSAR analysis of antitumor compounds of quinoacridinium derivatives calculated by the semi-empirical PM3 method

\begin{tabular}{|c|c|c|c|c|c|c|c|c|c|c|c|c|c|}
\hline \multirow{2}{*}{$\begin{array}{c}\text { Comp. } \\
\text { Number }\end{array}$} & \multicolumn{13}{|c|}{ Atomic net charges (Coulomb) } \\
\hline & qC1 & qC2 & qC3 & qC4 & qC5 & qC6 & qC7 & qN8 & qC9 & qC10 & qC11 & qC12 & qN13 \\
\hline 12 & -0.032 & -0.091 & 0.103 & -0.136 & -0.147 & 0.024 & -0.182 & 0.188 & -0.152 & -0.062 & .059 & -0.113 & 0.527 \\
\hline 13 & & & -0.112 & & & & & & & -0.030 & & .045 & \\
\hline 14 & 4 & -0.011 & -0.132 & & & & & 37 & -0.173 & -0.032 & & -0.057 & 0.418 \\
\hline 15 & & -0.083 & -0.096 & -0.105 & -0.142 & -0.014 & & 22 & -0.169 & -0.029 & -0.123 & -0.045 & 0.577 \\
\hline \multirow{2}{*}{$\begin{array}{c}\text { Comp. } \\
\text { Number }\end{array}$} & \multicolumn{8}{|c|}{ Atomic net charges (Coulomb) } & & E Eumo & & & \\
\hline & qC14 & qC15 & qC16 & qC17 & qC18 & qC19 & qC20 & qC21 & & (ev) & (ev) & $\left(\AA^{3}\right)$ & \\
\hline 12 & -0.184 & -0.006 & 0.047 & 0.013 & -0.126 & -0.104 & -0.045 & 0.001 & 17.825 & -2.485 & -8.947 & 41.64 & -0.57 \\
\hline 13 & & -0.076 & 0.072 & 0.011 & -0.1 & & & & 12.141 & -2.084 & & 42.12 & -0.44 \\
\hline 14 & 0.093 & -0.067 & 0.049 & -0.005 & -0.1 & -0.1 & -0.0 & -0.015 & 12.211 & -2.446 & -5.288 & 51.38 & 1.24 \\
\hline 15 & -0.128 & -0.020 & 0.053 & 0.005 & -0.112 & -0.120 & -0.025 & -0.010 & 13.966 & -2.229 & -5.155 & 42.70 & 1.05 \\
\hline
\end{tabular}

different electronic and molecular properties absolutely produce different antitumor activity.

Based on the above explanation and QSAR model equations (3), atomic charge values of qC3, qC4, qC9, qC11, qC12, qC14, $\mu$, ELumo, $\alpha$, and Log P for respective designed compounds are variables influencing the value of antitumor activity $\left(\mathrm{IC}_{50}\right)$ of quinoacridinium derivatives. In designing the molecule, it is necessary to consider the attachment of the atom or functional groups to the main framework of quinoacridinium derivatives which can cause the change of atomic charge value of qC3, qC4, qC9, qC11, qC12, qC14, $\mu$, Elumo, $\alpha$, and Log P, so that it can cause different predictions of $\mathrm{IC}_{50}$ value (Table V). The calculation of atomic charges and other descriptors obtained from each of the new compounds is incorporated into the QSAR model of equation (3) in order to obtain theoretical $\mathrm{IC}_{50}$ values of the novel compound derived from the modeling design. Compounds that have a small $\mathrm{IC}_{50}$ value are antitumor compounds that have the highest antitumor activity. The smaller $\mathrm{IC}_{50}$ value of a quinoacridinium derivative compound is, the higher the chances of the compound as an antitumor drug is and it may be proposed for synthesis in the laboratory.

The calculated IC50value of new molecules from the quinoacridinium derivatives design (Table VI and VII). Some compounds from quinoacridinium (Table VI and VII) derivative design are proposed for synthesis, in which they have an $\mathrm{IC}_{50}$ value smaller than $\mathrm{IC}_{50}$ of fifteen quinoacridinium derivatives (Tables I and II). Thus the quinoacridinium derivatives compounds derived from the recommended modeling design are: compounds $16 \quad\left(\mathrm{IC}_{50}=0.000002 \mu \mathrm{M}\right), 19$ $\left(\mathrm{IC}_{50}=0.125555 \mu \mathrm{M}\right), 20 \quad\left(\mathrm{IC}_{50}=0.173970 \mu \mathrm{M}\right), 22$
$\left(\mathrm{IC}_{50}=0.127722 \mu \mathrm{M}\right), \quad 23 \quad\left(\mathrm{IC}_{50}=0.091970 \mu \mathrm{M}\right) \quad 24$ $\left(\mathrm{IC}_{50}=0.000118 \mu \mathrm{M}\right), 25 \quad\left(\mathrm{IC}_{50}=0.000398 \mu \mathrm{M}\right), 26$ $\left(\mathrm{IC}_{50}=0.018086 \mu \mathrm{M}\right), \quad 27 \quad\left(\mathrm{IC}_{50}=0.055728 \mu \mathrm{M}\right) \quad 28$ $\left(\mathrm{IC}_{50}=0.122898 \mu \mathrm{M}\right), \quad 30 \quad\left(\mathrm{IC}_{50}=0.034668 \mu \mathrm{M}\right) \quad 31$ $\left(\mathrm{IC}_{50}=0.000004 \mu \mathrm{M}\right), 32 \quad\left(\mathrm{IC}_{50}=0.017990 \mu \mathrm{M}\right), 39$ $\left(\mathrm{IC}_{50}=0.040617 \mu \mathrm{M}\right)$, and $40\left(\mathrm{IC}_{50}=0.156537 \mu \mathrm{M}\right)$. Theoretically, they can be proposed for synthesis in the laboratory. Certainly, in the synthesis process in the laboratory, it is prioritized to synthesize compounds that have smaller $\mathrm{IC}_{50}$ values and compounds that have the easy synthesis pathways in the laboratory, as well as the availability of chemicals (Hadanu and Syamsudin, 2013; Hadanu et al., 2015). The theoretical $\mathrm{IC}_{50}$ value of the quinoacridinium derivatives is less than the $\mathrm{IC}_{50}$ value determined by the National Cancer Institute (NCI) for the compounds extracted from the natural material. According to the National Cancer Institute (NCI), an extract is considered to have an active anticancer activity if its $\mathrm{IC}_{50}$ value is $<30$ $\mu \mathrm{g} / \mathrm{mL}$, moderate active anticancer activity if its IC 50 value is $\geq 30 \mu \mathrm{g} / \mathrm{mL}, \mathrm{IC}_{50}<100 \mu \mathrm{g} / \mathrm{mL}$, and inactive anticancer activity if its $\mathrm{IC}_{50}$ value is $>100$ $\mu \mathrm{g} / \mathrm{mL}^{2}$

Based on the structure model of the QSAR analysis recommended for synthesis in the laboratory, it can be concluded that the quinoacridinium derivatives which have high and potential antitumor activities are quinoacridinium derivatives which have functional groups as follows: $-\mathrm{Cl}$ is bound to $\mathrm{C} 3$ and $\mathrm{C} 11$ atoms; the $-\mathrm{CH}_{3}$ function group is bound to $\mathrm{C} 6, \mathrm{C} 8$, and $\mathrm{C} 13$ atoms, anion $\mathrm{H}_{3} \mathrm{COSO}_{3} ;-\mathrm{OCH}_{3}$ functional group is bound to $\mathrm{C} 6$ atom, anion iodide $\left(\mathrm{I}^{-}\right)$; functional group of $\mathrm{CH}=\mathrm{CHCON}\left(\mathrm{CH}_{2} \mathrm{CH}_{2}\right)_{2} \mathrm{O}$ is bound to $\mathrm{C} 6$ and $\mathrm{C} 10$ atoms; the functional group of- $\left(\mathrm{CH}_{2}\right)_{3} \mathrm{OCOCH}_{3}$ is bound to $\mathrm{C} 3, \mathrm{C} 6$, and $\mathrm{C} 10 ;-\mathrm{C}_{2} \mathrm{H}_{5}$ functional group is 
bound to $\mathrm{C} 13$ atom, anion $\mathrm{H}_{5} \mathrm{C}_{2} \mathrm{OSO}_{3}$. Those all are bound to the main framework of quinoacridinium compounds. Nearly all compounds modeled according to Lipinski's Rule are quinoacridinium derivative compounds having fewer than 5 hydrogen bond donors (nitrogen or oxygen atoms with one or more hydrogen atoms), less than 10 hydrogen bond acceptors (nitrogen or oxygen atoms), and having an octanol-water partition coefficient ( $\log P$ ) is less than 5 .

\section{CONCLUSION}

Based on the results and discussion, it can be concluded that the best model of QSAR equation which shows the relationship between antitumor activity and latent variables to 11 compounds of quinoacridinium derivatives is: $\log \mathrm{IC}_{50}=-13.010$ $+15.338(\mathrm{qC} 3)-4.31(\mathrm{qC} 4)-155.308(\mathrm{qC} 9)+$ $33.626(\mathrm{qC11})+26.626(\mathrm{qC} 12)+24.631(\mathrm{qC} 14)-$ $0.228(\mu)-0.621$ (Е Еumo) $-0.066(\alpha)+0.233($ Log P); $\mathrm{n}=11,(\mathrm{r})=1.00,\left(\mathrm{r}^{2}\right)=1.00, \mathrm{SE}=0$, and PRESS $=$ 0.003 . A good correlation was observed between the experimental and predicted values of the anticancer activity $(\mathrm{R}=0.993)$, which indicated the validity and quality of the QSAR model developed in this work. Therefore, we conclude that the descriptors studied (e.g., atomic charge values qC3, qC4, qC9, qC11, qC12, qC14, $\mu$, Elumo, $\alpha$, and Log P), which influenced the structural features of the quino-acridinium, can be used in tandem with other topological descriptors for the development of predictive QSAR models. The design of quinoacridinium derivative compounds which have high theoretical antitumor activity and are recommended for synthesizing in the laboratory are 16 of 28 compounds. Such designed compounds are $16,19,20,22$ to $28,30,32,39$, and 40 .

\section{REFERENCES}

Alam M., Khan A., Wadood A., Khan A., Bashir S., et al., 2016. Bioassay-guided isolation of sesquiterpene coumarins from Ferula narthex bioss: A new anticancer agent. Frontiers in Pharmacology, 7(FEB), 1-6. https://doi.org/10.3389/fphar.2016.00026

Bladt TT., Frisvad JC., Knudsen PB., and Larsen TO. 2013. Anticancer and antifungal compounds from Aspergillus, Penicillium and other filamentous fungi. Molecules (Vol. 18). https://doi.org/10.3390/molecules180911 338

Bradley CJ., Yabroff KR., Dahman B., Feuer EJ., Mariotto A., and Brown ML. 2008. Productivity costs of cancer mortality in the
United States: 2000-2020. J. Nat. Canc. Institute, 100(24), 1763-1770. https://doi.org/10.1093/jnci/djn384

Cheng MK., Modi C., Cookson JC., Hutchinson I., Heald RA., et al., 2008. Antitumor polycyclic acridines. 20.1 Search for DNA quadruplex binding selectivity in a series of 8,13dimethylquino[4,3,2-kl]acridinium salts: Telomere-targeted agents. J. Medicinal Chemistry, 51(4), 963-975. https://doi.org/10.1021/jm070587t

Cookson JC., Heald RA., and Stevens MFG., 2005. Antitumor polycyclic acridines. 17. Synthesis and pharmaceutical profiles of pentacyclic acridinium salts designed to destabilize telomeric integrity. J.Medicinal Chemistry, 48(23), 7198-7207. https://doi.org/10.1021/jm058031y

Deep A., Narasimhan B., Lim SM., Ramasamy K., Mishra RK., and Mani V., 2016. 4Thiazolidinone derivatives: Synthesis, antimicrobial, anticancer evaluation and QSAR studies. RSC Advances, 6(111), 109485-109494. https://doi.org/10.1039/c6ra23006g

Ferguson AM., Heritage T., Jonathon P., Pack SE., Phillips L., Rogan J. and Snaith PJ., 1997. EVA: A new theoretically based molecular descriptor for use in QSAR/QSPR analysis. J. Computer-Aided Molecular Design, 11(2), 143-152. https://doi.org/10.1023/A:100802630879 0

Florea AM. and Büsselberg D. 2011. Cisplatin as an anti-tumor drug: Cellular mechanisms of activity, drug resistance and induced side effects. Cancers, 3(1), 1351-1371. https://doi.org/10.3390/cancers3011351

Fugmann B., Steffan B., and Steglich W., 1984. Necatorone, An Alkaloidal Pigment From The Gilled Toadstool Lactarius Necator (Agaricales). Tetrahedron Letters, 25(33), 3575-3578.

Hadanu R., Idris S. and Sutapa IW. 2015. QSAR analysis of benzothiazole derivatives of antimalarial compounds based on AM1 semi-empirical method. IJC., 15(1), 86-92. https://doi.org/10.22146/ijc.21228

Hadanu R., and Syamsudin. 2013. Quantitative structure-activity relationship analysis of antimalarial compound of mangostin derivatives using regression linear approach. Asian J.Chem., 25(11), 6136-6140.

Hagan DJ., Chan D., Schwalbe H. and Stevens MFG. 
(1998). Antitumour polycyclic acridines. Part 3 . 1 A two-step conversion of 9azidoacridine to $7 \mathrm{H}$-pyrido [ $4,3,2-\mathrm{kl}$ ] acridines by Graebe - Ullmann thermolysis of substituted 9- ( 1,2 , 3-triazol-1-yl ) acridines, 915-923.

Hagan DJ., Giménez-Arnau E., Schwalbe CH. and Stevens MFG. 1997. Antitumour polycyclic acridines. Part 1. Synthesis of 7H-pyridoand $8 \mathrm{H}$-quino-[4,3,2-kl]acridines by GraebeUllmann thermolysis of 9-(1,2,3-triazol-1yl)acridines: Application of differential scanning calorimetry to predict optimum cyclisation conditions. J. Chemical Society Perkin Transactions 1, (18), 2739-2746. https://doi.org/10.1039/a702299i

Heliawati L., Kardinan A., Mayanti T. and Tjokronegoro R ati. 2015. Piceatanol: Anticancer compound from Gewang seed extract. J Applied Pharmaceutical Science, 5(1), 110-113. https://doi.org/10.7324/JAPS.2015.50119

Hosny MA., Radwan HA. and El-Sawi EA. 2012. Synthesis and anticancer activity of some new derivatives of coumarin and quinolinyl mercaptotriazoles. E-Journal of Chemistry, 9(4),

1737-1745. https://doi.org/10.1155/2012/365647

Julino M. and Stevens MFG. 1998. Antitumour polycyclic acridines. Part 5.1Synthesis of 7Hpyrido[4,32-kl]acridines with exploitable functionality in the pyridine ring. $J$ the Chemical Society - Perkin Transactions 1, (10), 1677-1684. https://doi.org/10.1039/a800575c

Leonetti C. 2004. Biological Activity of the GQuadruplex Ligand RHPS4 (3,11-Difluoro6,8,13-trimethyl-8H-quino[4,3,2-

$\mathrm{kl}$ ]acridinium methosulfate) Is Associated with Telomere Capping Alteration. Molecular Pharmacology, 66(5), 1138-1146. https://doi.org/10.1124/mol.104.001537

Luo Z-H., He S-Y., Chen B-Q., Shi Y-P., Liu Y-M., Li C.W., and Wang Q-S. (2012). Synthesis and in Vitro Antitumor Activity of 1,3,4-Oxadiazole Derivatives Based on Benzisoselenazolone. Chemical \& Pharmaceutical Bulletin, 60(7), 887-891. https://doi.org/10.1248/cpb.c1200250

Miladiyah I., Tahir I., Jumina J., Mubarika S. Mustofa M. 2016. Quantitative Structure-Activity Relationship Analysis of Xanthone Derivates as Cytotoxic Agents in Liver Cancer Cell Line HepG2. Molekul, 11(1), 143. https://doi.org/10.20884/1.jm.2016.11.1.2 03

Missailidis S., Stanslas J., Modi C., Ellis MJ., Robins R. A., Laughton CA. and Stevens MFG. 2002. Antitumor Polycyclic Acridines . Part 12 . 1 Physical and Biological Properties A Lead Compound in Anticancer Drug Design, 13, 175-189.

Mota LF., Gaudio AC. and Takahata Y., 2006. Quantitative Structure-Activity Relationships of a Series of Chalcone Derivatives (1,3-Diphenyl-2-propen-1one) as Anti Plasmodium falciparum Agents (Anti Malaria Agents). Internet Electronic Journal of Molecular Design, 5(12), 555-569, https://doi.org/10.1103/PhysRevLett.104. 207002

Noolvi MN. and Patel HM. 2013. Synthesis, method optimization, anticancer activity of 2,3,7trisubstituted Quinazoline derivatives and targeting EGFR-tyrosine kinase by rational approach. 1st Cancer Update. Arabian Journal of Chemistry, 6(1), 35-48. https://doi.org/10.1016/j.arabjc.2010.12.0 31

Nugraha I., Annisa AN., Wibowo AT. and Kusuma A. M. 2018. Chemopreventive Activity of Kola (Cola Accuminata) Seed Ethanol Extract in Mice Induced by Cyclophosphamide. IOP Conference Series: Materials Science and Engineering, $288(1)$. https://doi.org/10.1088/1757899X/288/1/012008

Schmitt S. and Dou QP. 2013. Metal-Based Compounds as Proteasome-Inhibitory AntiCancer Drugs, 1(1), 1-3. https://doi.org/10.4172/.1000e101

Shelton J., Lu X., Hollenbaugh JA., Cho JH., Amblard, F. and Schinazi RF. 2016. Metabolism, Biochemical Actions, and Chemical Synthesis of Anticancer Nucleosides, Nucleotides, and Base Analogs. Chemical Reviews, 116(23), 14379-14455. https://doi.org/10.1021/acs.chemrev.6b00 209

Stanslas J., Hagan DJ., Ellis MJ., Turner C., Carmichael J., Ward W., et al., 2000. Antitumor polycyclic acridines. 7. Synthesis and biological properties of DNA affinic tetra- and pentacyclic acridines. Journal of Medicinal Chemistry, 43(8), 1563-1572. https://doi.org/10.1021/jm9909490

Su QG., Liu Y., Cai YC., Sun YL., Wang B. and Xian L. J. 2011. Anti-tumour effects of xanthone 
derivatives and the possible mechanisms of action. Investigational New Drugs, 29(6), 1230-1240.

https://doi.org/10.1007/s10637-0109468-5

Reddy TP. 2012. Exploring the Anti-inflammatory and Anti-cancer compounds \nfrom the leaves of Acalypha indica. IOSR Journal of Pharmacy and Biological Sciences (IOSRJPBS), 4(2), 01-07. Retrieved from http://www.iosrjournals.org/iosrjpbs/pages/v4i2.html
Torre LA., Bray F., Siegel RL., Ferlay J., LortetTieulent J. and Jemal A. 2015. Global Cancer Statistics, 2012. Cancer Statistics CA Cancer J Clin. https://doi.org/10.3322/caac.21262.

Tripodi F., Pagliarin R., Fumagalli G., Bigi A., Fusi P., Orsini F. et al., 2012. Synthesis and biological evaluation of 1,4-diaryl-2-azetidinones as specific anticancer agents: Activation of adenosine monophosphate activated protein kinase and induction of apoptosis. Journal of Medicinal Chemistry, 55(5), 21122124. https://doi.org/10.1021/jm201344a 